\title{
Repurposing Mebendazole as a Replacement for Vincristine for the Treatment of Brain Tumors
}

\author{
Michelle De Witt,,$^{1^{*}}$ Alexander Gamble, ${ }^{2^{*}}$ Derek Hanson, ${ }^{3^{*+}}$ Daniel Markowitz, ${ }^{1^{*}}$ Caitlin Powell, ${ }^{1+}$ \\ Saleh Al Dimassi, ${ }^{1}$ Mark Atlas, ${ }^{3,5}$ John Boockvar, ${ }^{1,2,5}$ Rosamaria Ruggieri, ${ }^{1,4,5}$ and Marc Symons ${ }^{1,2,5}$
}

${ }^{1}$ Karches Center for Oncology Research, The Feinstein Institute for Medical Research, Manhasset, New York, United States of America, and ${ }^{\dagger}$ current affiliation: Regeneron, Tarrytown, New York, United States of America; ${ }^{2}$ Department of Neurosurgery, Northwell Health, Manhasset, New York, United States of America; ${ }^{3}$ Division of Hematology-Oncology, Steven and Alexandra Cohen Children's Medical Center, Northwell Health, New Hyde Park, New York, United States of America, and ${ }^{+}$current affiliation: Joseph M. Sanzari Children's Hospital, Hackensack University Medical Center, Hackensack, New Jersey, United States of America; ${ }^{4}$ Department of Radiation Oncology, Northwell Health, and ${ }^{5}$ Hofstra Northwell School of Medicine, Hempstead, New York, United States of America

\begin{abstract}
The microtubule inhibitor vincristine is currently used to treat a variety of brain tumors, including low-grade glioma and anaplastic oligodendroglioma. Vincristine, however, does not penetrate well into brain tumor tissue, and moreover, it displays dose-limiting toxicities, including peripheral neuropathy. Mebendazole, a Food and Drug Administration-approved anthelmintic drug with a favorable safety profile, has recently been shown to display strong therapeutic efficacy in animal models of both glioma and medulloblastoma. Importantly, appropriate formulations of mebendazole yield therapeutically effective concentrations in the brain. Mebendazole has been shown to inhibit microtubule formation, but it is not known whether its potency against tumor cells is mediated by this inhibitory effect. To investigate this, we examined the effects of mebendazole on GL261 glioblastoma cell viability, microtubule polymerization and metaphase arrest, and found that the effective concentrations to inhibit these functions are very similar. In addition, using mebendazole as a seed for the National Cancer Institute (NCI) COMPARE program revealed that the top-scoring drugs were highly enriched in microtubule-targeting drugs. Taken together, these results indicate that the cell toxicity of mebendazole is indeed caused by inhibiting microtubule formation. We also compared the therapeutic efficacy of mebendazole and vincristine against GL261 orthotopic tumors. We found that mebendazole showed a significant increase in animal survival time, whereas vincristine, even at a dose close to its maximum tolerated dose, failed to show any efficacy. In conclusion, our results strongly support the clinical use of mebendazole as a replacement for vincristine for the treatment of brain tumors.
\end{abstract}

Online address: http://www.molmed.org

doi: 10.2119/molmed.2017.00011

\section{INTRODUCTION}

The microtubule polymerization inhibitor vincristine has a broad spectrum of use in cancer chemotherapy, for both hematological malignancies and solid tumors. Vincristine is also used in the treatment of a variety of central nervous system (CNS) tumors. Along with procarbazine and lomustine (CCNU), it constitutes the PCV regimen, which is currently considered standard of care for newly diagnosed patients with $1 \mathrm{p} / 19 \mathrm{q}$ co-deleted anaplastic oligodendroglioma $(1,2)$ and adult patients with low-grade glioma (3).

${ }^{*} M D W, A G, D H$, and DM contributed equally (in alphabetical order).

Address correspondence to Marc Symons, The Feinstein Institute for Medical Research, 350 Community Drive, Manhasset, NY 11030, USA. Phone: 516-562-1193; Fax: 516-562-1022; E-mail: msymons@northwell.edu

Submitted January 16, 2017; Accepted for Publication February 1, 2017;

Published Online (www.molmed.org) April 5, 2017.

Yyำ

Feinstein Institute

for Medical Research

Northwell Health-

Because of its large size (MW 825 daltons) and its susceptibility to transport by Mrp1 and Pgp, vincristine has poor blood-brain barrier $(\mathrm{BBB})$ penetrance $(4,5)$. Therefore, its use for the treatment of CNS tumors has been questioned $(4,6)$. Vincristine also has significant dose-limiting toxicities. The peripheral neuropathies resulting from its use can be debilitating and permanent, often halting use of the drug $(7,8)$.

Mebendazole, an anthelmintic with a rather benign side effect profile that has been in longstanding clinical use, has recently been shown to display significant therapeutic efficacy in animal models of both glioblastoma and medulloblastoma $(9,10)$. Depending on crystallization conditions, mebendazole can form three 
different polymorphs, termed A, B and C, which display significant differences in bioavailability $(11,12)$. As a result, the distinct polymorphs, when administered orally, also have different toxicity and therapeutic effects, as both anthelmintic and anticancer agents $(11,12)$. However, once dissolved, the respective polymorphs have identical properties. Both polymorphs $B$ and $C$ have good bioavailability and BBB penetration and display similar therapeutic efficacy in the GL261-C57BL/ 6 glioblastoma model, whereas polymorph A totally lacks in efficacy (12).

Mebendazole has been shown to inhibit microtubule formation $(9,13)$, but it is not known whether its tumor cell-killing effect is solely mediated by its microtubule destabilizing effect. Indeed, mebendazole has been shown to inhibit a number of protein kinases (14). In this study, therefore, we examined the mechanisms of tumor cell killing of mebendazole in detail, motivated by the possibility that mebendazole may be able to serve as a safer and more effective replacement for vincristine for the treatment of brain tumors. Thus, we determined the inhibitory effects of mebendazole on GL261 glioblastoma cell viability, metaphase arrest and microtubule polymerization and compared these effects with those of vincristine. We also compared the therapeutic efficacies and toxicities of mebendazole and vincristine in the GL261-C57BL/ 6 syngeneic orthotopic mouse model.

\section{MATERIALS AND METHODS}

\section{Tissue Culture}

The GL261 glioblastoma cell line was maintained in Dulbecco's Modified Eagle Medium (DMEM) supplemented with $10 \%$ fetal bovine serum (FBS) and antibiotics. All cells were maintained at $37^{\circ} \mathrm{C}$ in humidified air containing $5 \% \mathrm{CO}_{2}$.

\section{Cytotoxicity Assay}

GL261 cells were seeded in 96-well tissue culture plates at 3,000 cells per well. After $24 \mathrm{~h}$, cells were treated with a range of concentrations of either mebendazole or vincristine for $96 \mathrm{~h}$, with a medium change at $72 \mathrm{~h}$. At $96 \mathrm{~h}$, an MTT assay (Sigma) was performed and cell viability was determined by absorbance at $595 \mathrm{~nm}$.

\section{Tubulin Polymerization Assay}

The tubulin polymerization assay was performed essentially as described (15). In brief, GL261 glioblastoma cells were seeded at $3 \times 10^{5}$ cells per well in 6-well tissue culture plates. The next day, cells were treated with a range of concentrations of either mebendazole or vincristine for $24 \mathrm{~h}$. Subsequently, the cells were rinsed in PBS at $22^{\circ} \mathrm{C}$ and harvested at the same temperature in hypotonic lysis buffer ( $1 \mathrm{mM} \mathrm{MgCl}_{2}, 2 \mathrm{mM}$ EGTA, 0.5\% NP-40, 20 mM Tris $\mathrm{HCl} \mathrm{pH} 6.8$ ) containing protease inhibitors and benzonase. The samples were centrifuged at $13,000 \mathrm{~g}$ for $10 \mathrm{~min}$ at $22^{\circ} \mathrm{C}$ to separate polymerized (P) from soluble (S) tubulin. The supernatant containing the depolymerized (S) tubulin was transferred to a new tube. The pellet containing polymerized $(\mathrm{P})$ tubulin was resuspended in an equal amount of hypotonic lysis buffer. Equal amounts of protein were loaded on sodium dodecyl sulfate polyacrylamide gel electrophoresis (SDS-PAGE) gels and analyzed by western blotting with anti- $\alpha$-tubulin and GAPDH antibodies using chemiluminescence (Amersham ECL Western Blotting System, GE Healthcare). The blots were scanned and quantified using ImageJ 1.44p (National Institutes of Health). Microtubule polymerization was determined as the percentage of polymerized tubulin over total tubulin.

\section{Immunofluorescence}

GL261 cells were seeded at a density of 30,000 cells per well in 24-well plates containing coverslips coated with $0.1 \mathrm{mg} / \mathrm{mL}$ high-molecular-weight polylysine (Sigma) and subsequently with $0.5 \mu \mathrm{g} / \mathrm{cm}^{2}$ laminin from Engelbreth-Holm-Swarm murine sarcoma (Sigma). The following day, cells were exposed to $6 \mathrm{~Gy}$ of $\mathrm{X}$-ray radiation. At designated time points post-IR, cells were washed in PBS and fixed in $4 \%$ formaldehyde in PBS at room temperature. Once the cells were fixed, immunofluorescence was performed using an MPM2 antibody (Millipore) and counterstained with DAPI. For each condition, a total of 10 fluorescence micrograph images were taken with a Zeiss Axiovert 200M inverted microscope running on Axiovision software. For each image field, the total number of cells and the number of MPM2-positive cells were quantified. The mitotic index was calculated at each time point using the number of MPM2-positive cells as a percentage of the total number of cells counted in all 10 fields.

\section{Luciferase Expression by Lentivirus}

The pLenti-UBC-Gluc-T2A-Puro lentiplasmid was purchased from Targeting Systems. This plasmid was transfected into 293NT cells, along with plasmids carrying the virus reverse transcriptase gene $(R E V)$, the envelope glycoprotein gene ( $V S V-G$ ) and the polymerase gene (GAG-Pol), using Lipofectamine 2000 (Invitrogen). Virus was harvested after $48 \mathrm{~h}$ and used to infect GL261 cells to make GL261/GLuc.

\section{Animal Experiments}

Female C57BL/ 6 mice (age 3 months) were purchased from Jackson Labs. The transduced GL261/GLuc cells were used for brain tumor implantation. Tumor cells $(20,000 / 2 \mu \mathrm{L})$ were implanted using a stereotactic frame through a burr hole drilled $2 \mathrm{~mm}$ lateral to the bregma and $1 \mathrm{~mm}$ posterior to the coronal suture at a depth of $3 \mathrm{~mm}$ below the dura at a rate of $1 \mu \mathrm{L} / \mathrm{min}$.

Tail vein blood was collected daily and used for Gaussia luciferase quantification by luminometry assay as previously described (16). Gaussia luciferase substrate was purchased through Targeting Systems. Mice were randomly assigned to four different treatment groups once their G-luciferase values exceeded 1.3. Mebendazole was administered by oral gavage. Vincristine was administered 
by intraperitoneal injection. Polymorph C mebendazole tablets (S2017, Aurochem Laboratories Ltd.) were a gift from G. Riggins, Johns Hopkins University School of Medicine. Tablets were grounded into powder and resuspended in a PBS/sesame oil $(50 \% \mathrm{v} / \mathrm{v})$ mixture to achieve better gastrointestinal absorption of the drug. The gavage tip was coated with agave syrup prior to administration. Mebendazole suspensions were made fresh weekly.

Animals were observed daily for any signs of deterioration, neurotoxicity, movement disorders or signs of pain and distress in accordance with our Institutional Animal Care and Use Committee protocols, and euthanized when symptoms appeared.

\section{Von Frey Filament Testing}

Allodynia was determined essentially as described (17). Briefly, the mouse plantar surface of the hind paw was probed with calibrated von Frey hairs, with bending forces from $8 \mathrm{mg}$ to $4 \mathrm{~g}$. The 50\% withdrawal threshold (the force of the von Frey hair, in grams, to which the animal reacts in $50 \%$ of instances) was determined by applying the hairs six times. Time between measurements was at least $30 \mathrm{~s}$ between alternating paws. Comparison to baseline sensitivity was used to determine significant decrease in the withdrawal threshold.

\section{Statistical Analysis}

Direct comparisons were conducted using unpaired two-tailed Student $t$ test. Statistical analysis was performed using GraphPad Prism 6 Software. A P value lower than 0.05 was considered significant. The Kaplan-Meier estimate and a log-rank (Mantel-Cox) test were used to generate survival curves with the GraphPad Prism 6.0 software.

\section{RESULTS}

\section{Mebendazole and Vincristine Have Similar Cellular Mechanisms of Action}

To examine whether the cytotoxic effects of mebendazole are indeed caused by its inhibitory effect on microtubule formation, we compared the relative potencies of mebendazole and vincristine on microtubule formation and cell viability in GL261 glioma cells. Microtubule formation was quantified by hypotonic lysis of cells, followed by separation of polymerized and unpolymerized tubulin in the pellet and supernatant fractions, respectively (15). Cell viability was determined using the MTT assay.

From the data shown in Figure 1, we determined that the half maximal effective concentration $\left(\mathrm{EC}_{50}\right)$ of mebendazole for microtubule depolymerization (132 nM) is very similar to that for cell viability $(160 \mathrm{nM})$. A similar relationship holds for vincristine, although the difference between the $\mathrm{EC}_{50}$ for microtubule depolymerization (1.36 nM) and that for cell viability (2 $\mathrm{nM})$ is slightly larger.

As noted previously (15), the cellular microtubule polymerization assay

A

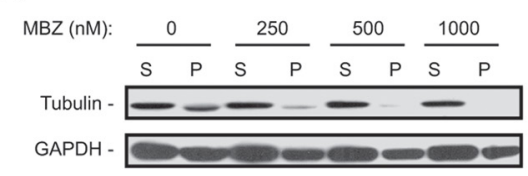

C

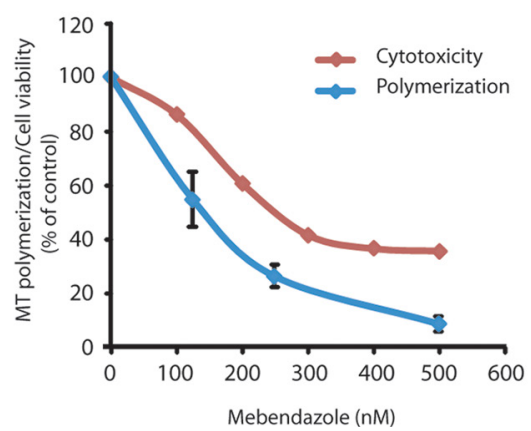

is subject to considerable variability. Therefore, to further examine the correlation between the effects of mebendazole on cytotoxicity microtubule depolymerization, we also determined the $\mathrm{EC}_{50}$ of both mebendazole and vincristine to induce mitotic arrest, a process that is critically dependent on microtubule formation. To determine the fraction of cells that are in the mitotic phase of the cell cycle, we used the MPM2 monoclonal antibody that binds to a phosphoamino acid-containing epitope present on multiple proteins during mitosis (18). Using this method, we first determined the time dependence of mitotic cell accumulation using a saturating concentration (500 nM) of mebendazole. We found that upon addition of mebendazole, the number of mitotic cells steadily increased over the first $8 \mathrm{~h}$ and reached a plateau by $24 \mathrm{~h}$ (Figure 2A). Next, using an incu-

B

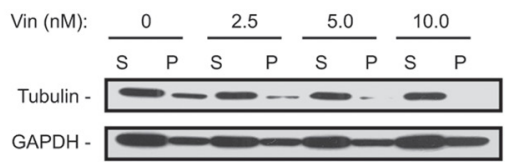

D

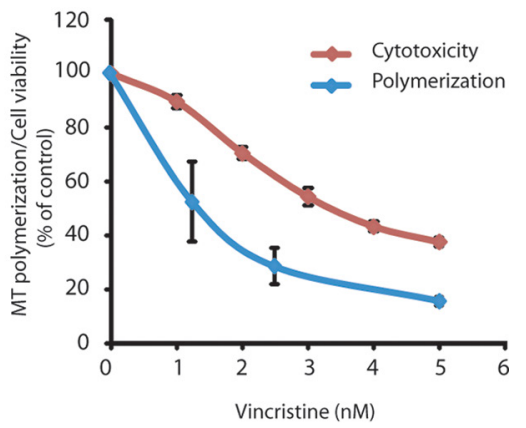

Figure 1. Mebendazole and vincristine have similar inhibitory effects on cell viability and microtubule polymerization. $(A, B)$ Western blots illustrating microtubule depolymerization caused by incubation of GL261 cells with the indicated concentrations of mebendazole (MBZ) and vincristine (VCR). Cells were processed, and polymerized (P) and soluble (S) tubulin were separated by centrifugation as described in Materials and Methods. $(C, D)$ Concentration dependence of microtubule polymerization and cell viability: (C) mebendazole, (D) vincristine. Microtubule polymerization was determined as the percentage of polymerized tubulin over total tubulin. Determination of cell viability was performed as described in Materials and Methods. Data shown were normalized to vehicle control values and represent the mean \pm SEM of three and at least eight independent experiments for the microtubule polymerization and cell viability experiments, respectively. 
A

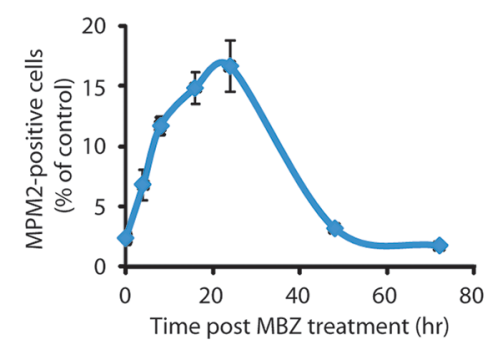

B

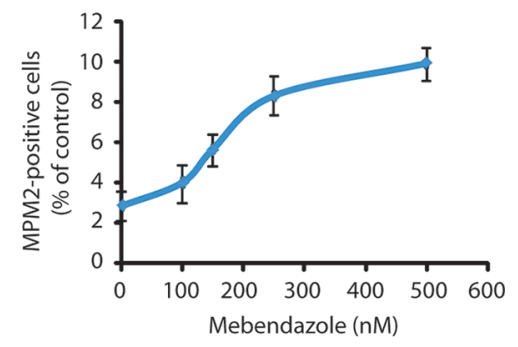

C

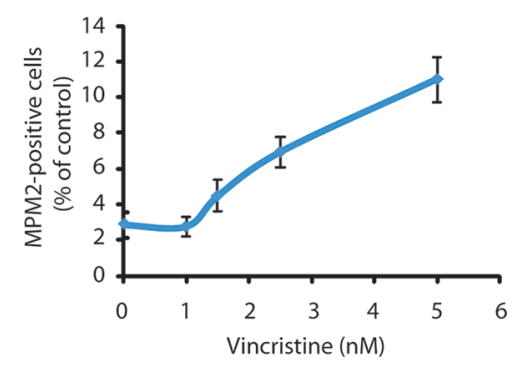

Figure 2. Mebendazole and vincristine induce metaphase arrest with a similar concentration dependence as that of cell viability. (A) Time dependence of mebendazole-induced metaphase arrest. Mebendazole concentration was $500 \mathrm{nM}$. Data shown represent the mean \pm SEM of three independent experiments. (B,C) Concentration dependence of metaphase arrest: (B) mebendazole,

(C) vincristine. Data shown represent the mean \pm SEM of three independent experiments.

bation time of $8 \mathrm{~h}$, we determined the respective $\mathrm{EC}_{50}$ values of mebendazole and vincristine for mitotic arrest to be $192 \mathrm{nM}$ and $2.49 \mathrm{nM}$ (Figures 2B, C).

Remarkably, the ratio of the $\mathrm{EC}_{50}$ for mitotic arrest over the $\mathrm{EC}_{50}$ for cell viability for mebendazole (1.20) is very similar to that for vincristine (1.24). Thus, the

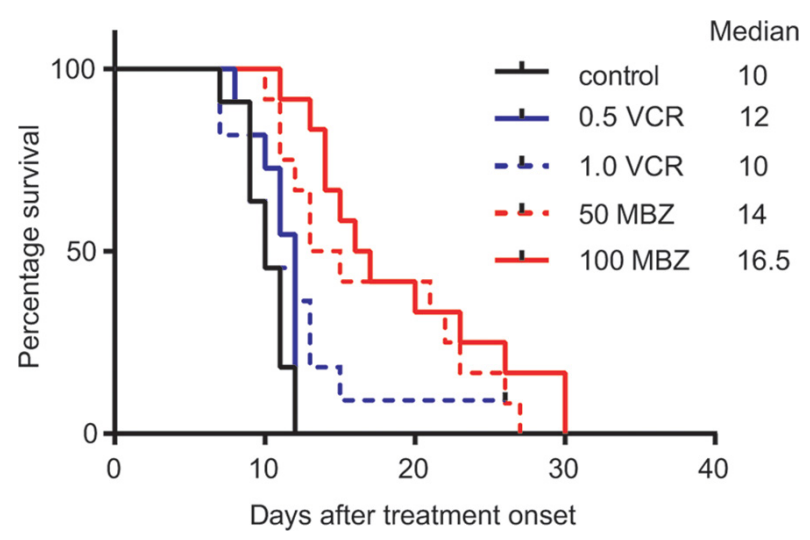

Figure 3. Mebendazole, but not vincristine, promotes the survival of glioma-bearing mice. Kaplan-Meier survival curves of mice implanted with GL261 glioma cells and treated with different doses of mebendazole and vincristine. Group sizes of the respective groups are 11-12.

data obtained with this more accurate method of probing cellular microtubule function strongly support the hypothesis that the cellular mechanisms of action of mebendazole and vincristine are indeed identical.

To further probe the mechanism of action of mebendazole, we used the COMPARE search tool of the NCI Developmental Therapeutics Program to find drugs that have similar mechanisms of toxicity in a set of 59 different human tumor cell lines that cover 9 different tissue types, including brain $(19,20)$. Of the 20 highest-scoring compounds with known mechanisms of action, $70 \%$ have been shown to modulate microtubule dynamics, including vinblastine, taxol, colchicine and lesser known drugs such as rhizoxin, podophyllotoxin and maytansine. Thus, this analysis provides further evidence that the major mechanism of action of mebendazole is microtubule destabilization.

\section{Mebendazole, but Not Vincristine, Promotes the Survival of Glioma- Bearing Mice}

With regard to therapeutic efficacy, it is important to note that, depending on crystallization conditions, mebendazole can form three different polymorphs, termed A, B and C, which display significant differences in solubility and bioavailability $(11,12)$. As a result, the distinct polymorphs, when administered orally, also have different toxicity and therapeutic effects, as both anthelmintic and anticancer agents $(11,12)$. However, once dissolved, the respective polymorphs have identical properties. As has been shown recently, polymorphs B and $C$ display similar therapeutic efficacy in the GL261-C57BL/6 model, with polymorph $C$ being slightly more effective than polymorph $\mathrm{B}$, and polymorph $\mathrm{A}$ totally lacking in efficacy (12). In this study, therefore, for all in vivo experiments, we used polymorph C.

For the animal studies, we performed orthotopic implantation of the GL261 cells in syngeneic C57BL/6 mice. To monitor tumor formation, we first transduced GL261 cells with Gaussia luciferase (Gluc), a secreted luciferase that can be quantified using a small volume of blood (16). Tail vein blood was collected every 1-3 d, and mice were randomized and treatments started once Gluc levels reached a predetermined value that corresponded to approximately $10 \mathrm{~d}$ after tumor cell implantation, as determined from pilot studies.

To determine the relative therapeutic efficacies of mebendazole and vincristine in mice, we used two doses for each drug. In the literature, values for the maximum 
tolerated dose (MTD) of vincristine for mice range from 1.17 to $1.5 \mathrm{mg} / \mathrm{kg}$ (21-23). Therefore, we chose to examine the effects of 0.5 and $1.0 \mathrm{mg} / \mathrm{kg}$ of vincristine. Dosing for mebendazole is less extensively documented in the literature. In one study, daily oral administration of mebendazole at $50 \mathrm{mg} / \mathrm{kg}$ was shown to be well tolerated, whereas a dose of $100 \mathrm{mg} / \mathrm{kg}$ showed apparent toxicity, as indicated by weight loss (9). However, as our pilot experiments did not reveal any toxicity at the higher dose of $100 \mathrm{mg} / \mathrm{kg}$, we included both $50 \mathrm{mg} / \mathrm{kg}$ and $100 \mathrm{mg} / \mathrm{kg}$ in this study.

The control group had a mean survival of $10.1 \mathrm{~d}$ after treatment onset (95\% confidence interval [CI], 9.2-11, Figure 3). Weekly intraperitoneal administration of vincristine at either $0.5 \mathrm{mg} / \mathrm{kg}$ (mean survival of $11 \mathrm{~d}, 95 \% \mathrm{CI}, 10.16-11.8$ ) or $1.0 \mathrm{mg} / \mathrm{kg}$ (mean survival of $11.9 \mathrm{~d}$, 95\% CI, 8.9-14.9) did not show a significant survival benefit. Daily oral gavage of mebendazole, however, significantly enhanced animal survival with both $50 \mathrm{mg} / \mathrm{kg}$ (mean survival of $17 \mathrm{~d}$, 95\% CI, 13.4-20.6) and $100 \mathrm{mg} / \mathrm{kg}$ (mean survival of $19 \mathrm{~d}, 95 \% \mathrm{CI}, 15.3-22.9$ ).

Peripheral neuropathy is a major side effect of vincristine and other microtubule drugs in the clinic. Thus, to quantify drug toxicity, we also examined the emergence of sensory allodynia by measuring footpad sensitivity using von Frey filaments. As paw withdrawal may be modulated by the presence of brain tumors, we performed an additional study in non-tumor-bearing animals. As expected, vincristine caused a significant increase in footpad sensitivity (Figure 4A). Mebendazole caused a tendency toward increased footpad sensitivity, but this increase was not statistically significant. We also examined the toxicity of combined mebendazole and vincristine. Combined administration of both drugs caused rapid weight loss after less than $1 \mathrm{wk}$ (Figure 4B), triggering euthanasia of the animals. Combining mebendazole with vincristine also appeared to further increase the emergence of neuropathy. In contrast,

A
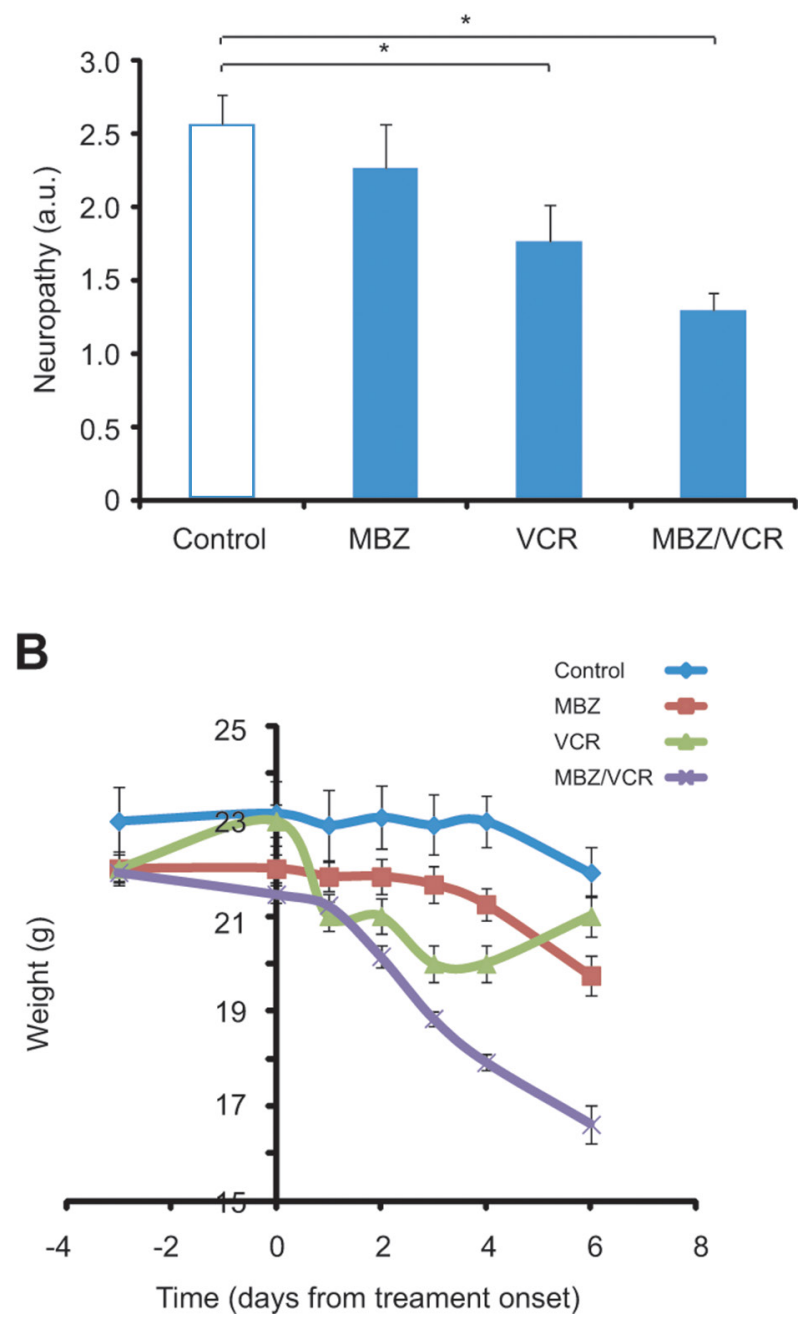

Figure 4. Drug-induced toxicities. (A) Peripheral neuropathy in mebendazole- and vincristinetreated mice. Shown are the mean $\pm \operatorname{SEM}(n=12)$ of footpad sensitivity measurements using von Frey filaments on d 6 after treatment initiation. (B) Animal weights before and during treatment. Data points represent the weight averages for each group $\pm \operatorname{SEM}(n=12)$.

administration of mebendazole or vincristine as monotherapy caused minor weight loss over this time period.

\section{DISCUSSION}

Our in vitro analysis strongly indicates that the mechanisms of cell killing by mebendazole are identical to those of vincristine. We also show that, whereas mebendazole has a significant therapeutic effect in the GL261-C57BL/6 model, vincristine has no efficacy at all in this model.
We have provided two independent lines of evidence indicating that the cytotoxicity of mebendazole is caused by its inhibitory effect on microtubule polymerization. First, the $\mathrm{EC}_{50}$ for mebendazole-induced cell toxicity is very similar to that of microtubule depolymerization and metaphase arrest. In addition, the same relationships between the $\mathrm{EC}_{50}$ values for mebendazole for cell toxicity, microtubule depolymerization and metaphase arrest hold for the effects of vincristine on these 
functions. Second, the NCI-DTP COMPARE analysis for cell toxicity revealed a high correlation between mebendazole and other microtubule inhibitors.

Mebendazole, at both doses examined, caused a significant extension of animal survival: $33 \%$ increase in survival after tumor cell implantation for mice treated with $50 \mathrm{mg} / \mathrm{kg}$ of mebendazole and $43 \%$ increase with $100 \mathrm{mg} / \mathrm{kg}$. We note that these therapeutic effects are not as robust as those obtained previously in the same model $(63 \%$ increase in survival for mice treated with $50 \mathrm{mg} / \mathrm{kg}$ of mebendazole). A major factor that likely contributes to the difference in therapeutic efficacy is that in our study, treatment was started much later $(10 \mathrm{~d})$ after tumor cell implantation than in the previous study (5 d) (24). In addition, the GL261 cells used in our study appear to be significantly more aggressive, as the control mice survived for a significantly shorter time, even though fewer tumor cells were implanted.

Mebendazole is often said to be well tolerated with few side effects $(25,26)$. However, at the higher doses and prolonged treatment periods necessary for the clinical management of severe echinococcosis infection, and likely also for its use as cancer chemotherapy, significant toxicities have been observed, including anemia, leukopenia and liver toxicity (27). We also note that the treatment of mice with both mebendazole and vincristine at doses close to their respective MTDs rapidly led to severe toxicity, which cautions against the combined use of these microtubule inhibitors in the clinic.

In contrast to mebendazole, we observed that vincristine, also at a dose close to its MTD, failed to show any therapeutic benefit in the GL261-C57BL/6 model in the conditions that we used. Most likely, this lack of efficacy is due to vincristine's poor BBB permeability $(4,5)$, in contrast to the good BBB permeability of mebendazole (12).

The extensive use of vincristine in brain tumor therapy (1-3) dates back to a phase II study using a combination of procarbazine, CCNU and vincristine
(PCV) to treat a wide range of brain tumors (28). The introduction of this combination was based on the therapeutic activity of procarbazine in an intracerebral rat leukemia model (29) and that of CCNU in orthotopic models of glioma and ependymoblastoma (30). Including vincristine in this regimen was based on limited clinical experience in a small number of patients $(31,32)$.

\section{CONCLUSION}

Our findings documenting a lack of therapeutic efficacy of vincristine in a mouse model for glioma where mebendazole shows significant efficacy, in addition to a lack of evidence for the clinical benefit of vincristine in the treatment of brain tumors, strongly suggest that vincristine should not be used chemotherapeutically in neuro-oncology. Thus, as mebendazole and vincristine have the same mechanism of action, replacing vincristine with mebendazole may facilitate the elimination of vincristine from neuro-oncological regimens.

\section{ACKNOWLEDGEMENTS}

Mebendazole tablets were a generous gift from G. Riggins, Johns Hopkins University School of Medicine. This research was supported by grants to MS from the Swim Across America Foundation and the Project to Cure Foundation, to JB and MS from the Voices Against Brain Cancer Foundation and to RR from the Zankel Foundation.

\section{DISCLOSURE}

The authors declare they have no competing interests as defined by Molecular Medicine, or other interests that might be perceived to influence the results and discussion reported in this paper.

\section{REFERENCES}

1. van den Bent MJ et al. (2013) Adjuvant procarbazine, lomustine, and vincristine chemotherapy in newly diagnosed anaplastic oligodendroglioma: long-term follow-up of EORTC brain tumor group study 26951. J. Clin. Oncol. 31:344-50.
2. Cairncross G et al. (2013) Phase III trial of chemoradiotherapy for anaplastic oligodendroglioma: long-term results of RTOG 9402. J. Clin. Oncol. 31:337-43.

3. Buckner JC et al. (2016) Radiation plus Procarbazine, CCNU, and Vincristine in Low-Grade Glioma. N. Engl. J. Med. 374:1344-55.

4. Boyle FM, Eller SL, Grossman SA (2004) Penetration of intra-arterially administered vincristine in experimental brain tumor. Neuro. Oncol. 6:300-05.

5. Wang F, Zhou F, Kruh GD, Gallo JM (2010) Influence of blood-brain barrier efflux pumps on the distribution of vincristine in brain and brain tumors. Neuro. Oncol. 12:1043-9.

6. Ajaz M, Jefferies S, Brazil L, Watts C, Chalmers A (2014) Current and investigational drug strategies for glioblastoma. Clin. Oncol. (R. Coll. Radiol.) 26:419-30.

7. Gidding CE, Kellie SJ, Kamps WA, de Graaf SS (1999) Vincristine revisited. Crit. Rev. Oncol. Hematol. 29:267-87.

8. Moore A, Pinkerton R (2009) Vincristine: Can its therapeutic index be enhanced? Pediatr. Blood Cancer. 53:1180-7.

9. Bai RY, Staedtke V, Aprhys CM, Gallia GL, Riggins GJ (2011) Antiparasitic mebendazole shows survival benefit in 2 preclinical models of glioblastoma multiforme. Neuro. Oncol. 13:974-82.

10. Bai RY, Staedtke V, Rudin CM, Bunz F, Riggins GJ (2015) Effective treatment of diverse medulloblastoma models with mebendazole and its impact on tumor angiogenesis. Neuro. Oncol. 17:545-54.

11. Rodriguez-Caabeiro F et al. (1987) Experimental chemotherapy and toxicity in mice of three mebendazole polymorphic forms. Chemotherapy. 33:266-71.

12. Bai RY et al. (2015) Brain Penetration and Efficacy of Different Mebendazole Polymorphs in a Mouse Brain Tumor Model. Clin. Cancer Res. 21:3462-70.

13. Sasaki J et al. (2002) The anthelmintic drug mebendazole induces mitotic arrest and apoptosis by depolymerizing tubulin in non-small cell lung cancer cells. Mol. Cancer Ther. 1:1201-9.

14. Issa NT, Peters OJ, Byers SW, Dakshanamurthy S (2015) RepurposeVS: A Drug Repurposing-Focused Computational Method for Accurate Drug-Target Signature Predictions. Comb. Chem. High Throughput Screen. 18:784-94.

15. Poruchynsky MS et al. (2004) Tumor cells resistant to a microtubule-depolymerizing hemiasterlin analogue, HTI-286, have mutations in alpha- or beta-tubulin and increased microtubule stability. Biochemistry. 43:13944-54.

16. Wurdinger T et al. (2008) A secreted luciferase for ex vivo monitoring of in vivo processes. Nat. Methods. 5:171-3.

17. Hansen N et al. (2011) Serotonin transporter deficiency protects mice from mechanical allodynia and heat hyperalgesia in vincristine neuropathy. Neurosci. Lett. 495:93-7. 
18. Sheen JH, Woo JK, Dickson RB (2003) c-Myc alters the DNA damage-induced G2/M arrest in human mammary epithelial cells. Br. J. Cancer. 89:1479-85.

19. Paull KD et al. (1989) Display and analysis of patterns of differential activity of drugs against human tumor cell lines: development of mean graph and COMPARE algorithm. J. Natl. Cancer Inst. 81:1088-92.

20. Holbeck SL, Collins JM, Doroshow JH (2010) Analysis of Food and Drug Administration-approved anticancer agents in the NCI60 panel of human tumor cell lines. Mol. Cancer Ther. 9:1451-60.

21. Kai K et al. (2006) Species and sex differences in susceptibility to olfactory lesions among the mouse, rat and monkey following an intravenous injection of vincristine sulphate. Toxicol. Pathol. 34:223-31.

22. Thompson J et al. (1999) Synergy of topotecan in combination with vincristine for treatment of pediatric solid tumor xenografts. Clin. Cancer Res. 5:3617-31.

23. Silverman JA, Deitcher SR (2013) Marqibo(R) (vincristine sulfate liposome injection) improves the pharmacokinetics and pharmacodynamics of vincristine. Cancer Chemother. Pharmacol. 71:555-64.

24. Bai RY, Staedtke V, Aprhys CM, Gallia GL, Riggins GJ (2011) Antiparasitic mebendazole shows survival benefit in 2 preclinical models of glioblastoma multiforme. Neuro Oncol. 13:974-82.

25. Vuitton DA (2009) Benzimidazoles for the treatment of cystic and alveolar echinococcosis: what is the consensus? Expert Rev. Anti. Infect. Ther. 7:145-9.

26. Pantziarka P, Bouche G, Meheus L, Sukhatme V, Sukhatme VP (2014) Repurposing Drugs in Oncology (ReDO): mebendazole as an anti-cancer agent. Ecancermedicalscience. 8:443.

27. Davis A, Dixon H, Pawlowski ZS (1989) Multicentre clinical trials of benzimidazole-carbamates in human cystic echinococcosis (phase 2). Bull. World Health Organ. 67:503-8.

28. Gutin PH et al. (1975) Phase II study of procarbazine, CCNU, and vincristine combination chemotherapy in the treatment of malignant brain tumors. Cancer. 35:1398-404.

29. Grunberg E (1970) Experimental tumor inhibitory activity of procarbazine. In: Carter SK, ed. Proceedings of the Chemotherapy Conference on Procarbazine. Bethesda, MD: US Govt. Printing Office, pp. 9-17.

30. Shapiro WR (1971) Studies on the chemotherapy of experimental brain tumors: evaluation of 1 (2-chloroethyl)-3-cyclohexyl-1-nitrosourea, vincristine, and 5-fluorouracil. J. Natl. Cancer Inst. 46:359-68.

31. Lassman LP, Pearce GW, Gang J (1965) Sensitivity of intracranial gliomas to vincristine sulphate. Lancet. 1:296-98.

32. Smart CR et al. (1968) Clinical experience with vincristine (NSC-67574) in tumors of the central nervous system and other malignant diseases. Cancer Chemother. Rep. 52:733-41.
Cite this article as: De Witt M, et al. (2017) Repurposing mebendazole as a replacement for vincristine for the treatment of brain tumors Mol. Med. 23:50-6. 